\title{
ISOLAMENTO E AVALIAÇÃO DA ATIVIDADE CITOTÓXICA DE ALGUNS ALCALÓIDES OXAPORFÍNICOS OBTIDOS DE ANNONACEAE
}

Denise Brentan da Silva, Maria de Fátima Cepa Matos, Simone T. Nakashita, Carina K. Misu, Nídia Cristiane Yoshida, Carlos Alexandre Carollo, João Roberto Fabri, Hércules da Silva Miglio e João Máximo de Siqueira*

Departamento de Farmácia, Centro de Ciencias Biológicas e da Saúde, Universidade Federal de Mato Grosso do Sul, CP 549, 79070-900 Campo Grande - MS, Brasil

Recebido em 23/6/06; aceito em 18/5/07; publicado na web em 25/10/07

\begin{abstract}
ISOLATION AND CYTOTOXICITY EVALUATION OF SOME OXOAPORPHINE ALKALOIDS FROM ANNONACEAE. A different methodology was used to isolate and purify oxoaporphine alkaloids, as they are difficult to separate by the usual workup when in mixture. Alkaloid extracts from Annonaceae species were obtained by base/acid extraction. The extracts were concentrated and submitted to partition in solutions of acids of different pKa values, followed by separation by preparative TLC using $1 \mathrm{~mm}$ thick silica gel impregnated with oxalic acid $(11.2 \% \mathrm{w} / \mathrm{w})$. Liriodenine, lisycamine, lanuginosine, and $O$-methylmoschatoline were obtained and tested against tumoral cells (line $\mathrm{Hep}_{2}$, ATCC-CCL 23, larynx carcinoma). Only $O$-methylmoschatoline $\left(\mathrm{IC}_{50} 12.4 \mu \mathrm{M}\right)$ was more active than cisplatin $(18.0 \mu \mathrm{M})$.
\end{abstract}

Keywords: oxoaporphine; chromatography; cytotoxicity.

\section{INTRODUÇÃO}

A classe dos alcalóides aporfínicos, ou aporfinóides, derivados do esqueleto isoquinolínico, é bastante destacada em diferentes espécies de Annonaceae ${ }^{1}$, sendo também encontrada em outras famílias². Esta classe está divida em diferentes subclasses, cujos representantes apresentam perfil estrutural característico (Figura 1) e, sem dúvida, propriedades químicas e biológicas próprias dos subgrupos a que perten$\mathrm{cem}^{3}$. Esses alcalóides caracterizam-se por apresentar esqueleto aporfínico (Figura 1a), e um de seus subgrupos, o oxaporfínico (Figura $1 b)$, possui esqueleto totalmente aromático e com presença de grupo carbonílico em C-7 ${ }^{1}$. Em função do padrão de substituição encontrado no anel, diferentes alcalóides oxaporfínicos já foram descritos.

Com relação às atividades biológicas e farmacológicas dos alcalóides oxaporfínicos, são conhecidas as seguintes: citotóxica ${ }^{1,2,4}$, anti-agregante plaquetária ${ }^{5}$, antibacteriana, antifúngica e a antiplasmódica ${ }^{6}$, ainda que esta última seja pouco significativa, quando comparada à da cloroquina. Dentre essas atividades, a mais representativa nessa subclasse é a citotóxica, que tem sido demonstrada frente a diferentes linhagens de células tumorais ${ }^{1,2}$. Este é o caso, por exemplo, da liriodenina, comumente isolada e, conseqüentemente, um dos alcalóides aporfínicos mais citados e testados ${ }^{2}$. A liriodenina foi usada como modelo comparativo com outros dois alcalóides aporfinóides — bulbocapnina e dicentrina — para explorar a relação entre estrutura e atividade inibidora sobre topoisomerase II. Várias características estruturais e propriedades físico-químicas são necessárias para avaliação da atividade farmacológica ${ }^{7}$ ou biológica, e a condição de que uma molécula seja totalmente planar não é um fator determinante. Foi sugerido, porém, que a planaridade desses alcalóides é um fator limitante para a atividade acima mencionada — ou seja, a liriodenina, por ser totalmente planar, seguida da dicentrina, que adota uma conformação relativamente planar, foram potentes inibidoras catalíticas de topoisomerase II, enquanto a bulbocapnina, que não apresenta tal conformação, mostrou-se inativa ${ }^{8}$.

*e-mail: jmaximo@nin.ufms.br

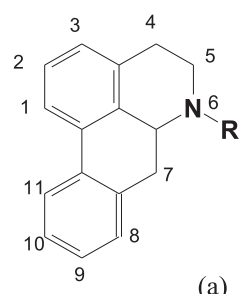

(a)

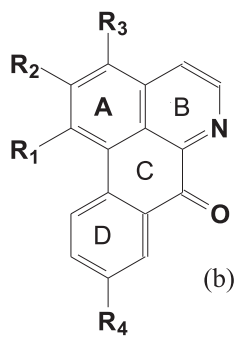

$\mathrm{R}_{1}+\mathrm{R}_{2}=\mathrm{OCH}_{2} \mathrm{O}, \mathrm{R}_{3}=\mathrm{R}_{4}=\mathrm{H}$, (1), liriodenina $\mathrm{R}_{1}+\mathrm{R}_{2}=\mathrm{OCH}_{2} \mathrm{O}, \mathrm{R}_{3}=\mathrm{H}, \mathrm{R}_{4}=\mathrm{OCH}_{3}$, (2), lanuginosina $\mathrm{R}_{1}+\mathrm{R}_{2}=\mathrm{OCH}_{2} \mathrm{O}, \mathrm{R}_{3}=\mathrm{R}_{4}=\mathrm{OCH}_{3}$, (3), oxobuxifolina $\mathrm{R}_{1}=\mathrm{R}_{2}=\mathrm{OCH}_{3}, \mathrm{R}_{3}=\mathrm{R}_{4}=\mathrm{H}$, (4), lisicamina $\mathrm{R}_{1}=\mathrm{R}_{2}=\mathrm{R}_{3}=\mathrm{OCH}_{3}, \mathrm{R}_{4}=\mathrm{H}$, (5), O-metilmoscatolina $\mathrm{R}_{1}+\mathrm{R}_{2}=\mathrm{OCH}_{2} \mathrm{O}, \mathrm{R}_{3}=\mathrm{OCH}_{3}, \mathrm{R}_{4}=\mathrm{H},(6)$, aterospermidina

Figura 1. Estrutura básica dos esqueletos de alcalóides aporfínicos (a) e oxaporfínicos $(b)$

Procedimentos para o isolamento e purificação dos alcalóides oxaporfínicos têm sido descritos e podem envolver várias etapas e/ ou requerer equipamentos menos acessíveis, tais como aparelhos de cromatografia líquida de média eficiência ${ }^{9}$ e de cromatografia líquida de alta eficiência (CLAE) $)^{10}$, com o decorrente alto custo para a realização. Geralmente, quando há dois ou mais representantes dessa classe de alcalóide presentes em uma mesma fração, mesmo que haja diferenças em seus padrões de substituição, enfrenta-se maior grau de dificuldade no isolamento e purificação ${ }^{11}$, pois geralmente observa-se o mesmo comportamento cromatográfico em métodos usuais de cromatografia (cromatografia em coluna com sílica ou alumina, cromatografia em camada analítica ou preparativa).

Com o intuito de aprofundar o estudo do comportamento dos alcalóides oxaporfínicos perante diferentes meios, complementá-lo com o estudo da atividade citotóxica e também contribuir com o conhecimento da relação estrutura-atividade, desenvolveu-se uma nova metodologia de baixo custo que se revelou eficiente no isola- 
mento e purificação desses compostos. Devido à utilização de placas cromatográficas preparativas de sílica-gel e impregnadas com ácido oxálico, foi possível separar misturas de dois ou mais alcalóides oxaporfínicos que seriam de difícil separação em placas usuais de sílica-gel: liriodenina (1) e lisicamina (4), de Unonopsis lindimani; lanuginosina (2), oxobuxifolina (3) e $O$-metilmoscatolina (5), de Duguetia glabriuscula; e $\mathbf{1}$ e $\mathbf{2}$ também de Duguetia furfuracea. Ainda com emprego de cromatografia em coluna de sílica-gel e utilizando eluentes aditivados com $\mathrm{HCl}$, realizou-se a separação de aterospermidina $(\mathbf{6})$ e $\mathbf{1}$.

A utilização de diferentes meios (suporte sólido e aditivado com ácido; solvente de eluição aditivado com ácido) proporcionou a separação desses representantes da classe de oxaporfínicos de acordo com seu comportamento em meio ácido. Foram utilizadas ferramentas de química teórica (Gaussian 03 e GaussView), visando a interpretação dos resultados experimentais obtidos no presente trabalho. Como esses alcalóides apresentam semelhanças estruturais, foram melhor exploradas outras propriedades como densidade de carga, potencial eletrostático e interação entre o par iônico, que auxiliaram no melhor entendimento dessa metodologia de separação.
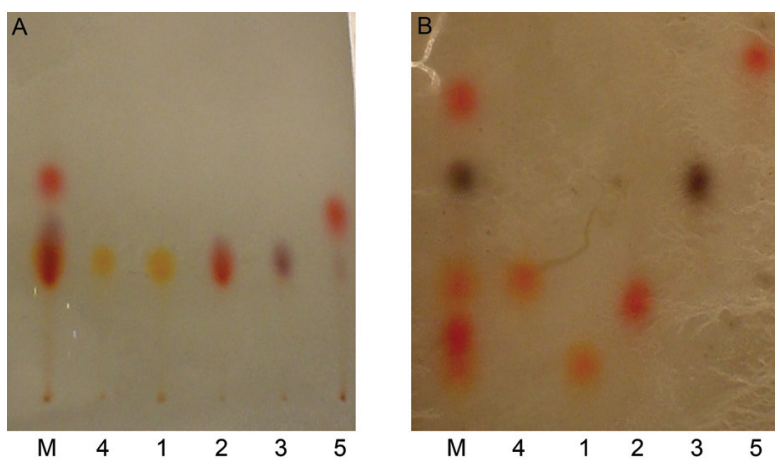

Figura 2. Comportamento dos alcalóides oxaporfínicos liriodenina (1), lanuginosina (2), oxobuxifolina (3), lisicamina (4) e O-metilmoscatolina (5) em suportes de: (A) sílica-gel, camada de 1,0 $\mathrm{mm}$ de espessura, revelador $\mathrm{HCl} 5 \%$ em EtOH, eluente $\mathrm{CHCl}_{3}: \mathrm{MeOH}$ a 2\%; (B) sílica-gel (1,0 mm de espessura) impregnada com ácido oxálico a $12,1 \%(\mathrm{~m} / \mathrm{m})$, eluente $\mathrm{CHCl}_{3}: \mathrm{MeOH}$ a $22 \%$, sem revelação

\section{RESULTADOS E DISCUSSÃO}

A utilização de suportes modificados e eluentes aditivados está bem documentada na literatura ${ }^{12}$. Bons exemplos são o uso de placas de sílica impregnadas com $\mathrm{AgNO}_{3}$ para separação de compostos com ligação dupla e a adição de dietilamina em eluentes para melhor resolução de extratos alcaloídicos em suportes com características ácidas. Recentemente, a utilização de agentes surfactantes foi proposta como uma metodologia mais rápida e de baixo custo para obtenção de extratos ricos em alcalóides ${ }^{13}$. Essas metodologias geralmente envolvem o conhecimento das propriedades físico-químicas do eluente, solvente e substância (ou extrato) envolvidos e as técnicas decerto visam a redução de custo, uma vez que metodologias mais eficientes são mais caras.

Durante os últimos anos temos trabalhado com alcalóides oxaporfínicos da família Annonaceae ${ }^{11}$, classe de compostos bastante comuns nessa família ${ }^{1}$, os quais apresentam amplo espectro de atividades biológicas e farmacológicas. Por outro lado, a estrutura totalmente planar e a baixa diversificação encontrada no padrão de substituição permitiriam justificar o comportamento semelhante de seus derivados frente a diferentes eluentes e suportes sólidos comumente utilizados (sílica e alumina).
De maneira geral, o comportamento do esqueleto alcaloídico em função do $\mathrm{pH}$ é bastante conhecido ${ }^{14}$ e geralmente é útil durante o procedimento usual de extração e purificação. Dependendo do esqueleto alcaloídico presente, tal procedimento pode compor-se de etapas específicas que podem se revelar etapas-chave do procedimento usual de extração e purificação ${ }^{14}$. Por analogia, as informações já descritas sobre o comportamento do esqueleto isoquinolínico em meio ácido diluído ou concentrado foram utilizadas como modelo: a população presente das espécies correspondentes às formas neutra e protonada deste esqueleto (isoquinolínico e isoquinolônio) é conhecida e dependente de $\mathrm{pH}^{15}$. Outra informação mais específica e referente ao esqueleto oxaporfínico é que este, em meio ácido, apresenta cor avermelhada bastante intensa, que decorre da protonação de N-6 e da deslocalização da carga positiva em todo o sistema aromático, causando um deslocamento batocrômico ${ }^{1}$. Com essas informações preliminares, foi possível avaliar o comportamento dos alcalóides oxaporfínicos em diferentes soluções ácidas.

Os extratos alcaloídicos foram obtidos pelo método usual ácido/base de três espécies vegetais da família Annonaceae: Duguetia furfuracea, D. glabriuscula e Unonopsis lindmanii. Após concentração a vácuo, foram solubilizados em clorofórmio e submetidos a partições sucessivas com soluções ácidas a 5\% de ácido oxálico (pKa 1,3), ácido cítrico (pKa 3,1) e ácido acético (pKa 4,7), observando-se uma maior proporção de alcalóides oxaporfínicos na fase aquosa mais ácida.

A partir desse resultado preliminar, foram então confeccionadas placas cromatográficas em camadas delgada e preparativa de sílica-gel impregnada com ácido acético, cítrico e oxálico. As placas impregnadas com ácido oxálico mostraram-se satisfatórias na separação dos alcalóides oxaporfínicos, mesmo nas misturas de substâncias que apresentam o mesmo fator de retenção em placas usuais de camadas delgada e preparativa com sílica-gel (Figura 2), resultando na separação dos seguintes alcalóides: lisicamina (4) e liriodenina (1), das cascas do caule de $U$. lindimanii; lanuginosina (2), oxobuxifolina (3) e $O$-metilmoscatolina (5), de uma mesma fração obtida das cascas do caule de D. glabriuscula, sendo que $\mathbf{5}$ foi isolado pela primeira vez nessa espécie vegetal; aterospermidina (6) e 1, foram separadas dos ramos aéreos de D. furfuracea, embora para esta espécie tenha sido utilizada cromatografia em coluna com gradiente de solvente $\left(\mathrm{CHCl}_{3}: \mathrm{MeOH}\right.$, aditivada com $0,5 \%$ de $\mathrm{HCl}$ ), sendo as estruturas elucidadas a partir de dados espectrais de RMN ${ }^{1} \mathrm{H}$ em comparação com os encontrados na literatura ${ }^{16}$.

O comportamento observado para esses alcalóides no suporte mais ácido foi muito similar ao observado para o modelo isoquinolínico já citado, no qual a maior população de espécie protonada pode ser encontrada em pH mais ácido, dessa forma favorecendo a retenção dos mesmos no suporte utilizado. A diferença no fator de retenção (Rf) entre os alcalóides oxaporfínicos 1-5, no suporte ácido (Figura 2), poderia ser função do padrão de substituição presente em cada representante. O padrão de substituição nas posições C-1, C-2 e C-3 do anel A contribui de maneira distinta para o valor de pKa e seria um fator determinante, se presente isoladamente. A inserção de um substituinte metoxila na posição C-2 poderia produzir, por exemplo, um incremento de 0,5 unidade de pKa, quando comparado ao pKa de um derivado com substituinte na posição C-1 ou C-3, ou mesmo comparado com derivados sem substituintes nessas posições ${ }^{17}$. Esse efeito sobre pKa, possivelmente ocorre pela contribuição na estabilização da carga, que o substituinte pode proporcionar. Todos os alcalóides apresentaram um padrão de substituição muito semelhante no anel A, e a variação que poderia ocorrer nessa contribuição seria em função do impedimento estérico entre os substituintes, uma vez que todas as substâncias analisadas apresentam dois ou mais substituintes nas posições C-1, C-2 e C-3. Os compostos 3 e $\mathbf{5}$ apresentam 
substituintes nas posições C-1, C-2 e C-3 e $\mathbf{4}$ as tem em C-1 e C-2, sendo alguns destes grupos volumosos $\left(-\mathrm{OCH}_{3}\right)$, o que dificultaria a coplanaridade do substituinte em $\mathrm{C}-2$ e, conseqüentemente, a contribuição dessa posição na estabilização da carga em N-6 seria menor. Já em 1 e 2, cujo substituinte nas posições C-1 e C-2 é um grupo metilenodioxila, a formação de um anel de cinco membros, que é mais rígido, favorece a coplanaridade.

\section{Parte teórica}

Com o estudo químico teórico das propriedades eletrônicas desses alcalóides foi possível obter informações que também possam contribuir para o esclarecimento do processo de separação em sílicagel impregnada com ácido oxálico, pois as diferenças mínimas observadas frente ao padrão de substituição, que dificultam o isolamento e purificação em suportes normais, podem afetar consideravelmente as propriedades eletrônicas. Com o pacote Gaussian 03 foi utilizado o método semi-empírico AM1 para uma primeira otimização. Posteriormente, para o refinamento dos cálculos, utilizou-se o ab initio DFT/B3LYP/6-31g**, para obtenção da densidade de carga total de $\mathbf{1}, \mathbf{2}, \mathbf{3}, \mathbf{4}$ e 5. Através das imagens geradas pelo pacote Hyperchem, com o qual se obteve uma visão qualitativa do tema (Figura 3), observa-se a participação eletrônica do par de elétrons do nitrogênio na ressonância do sistema anelar, o que reduziria a disponibilidade deste para protonação perante o ácido oxálico.

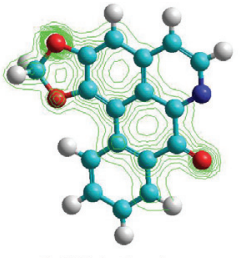

1. Liriodenina

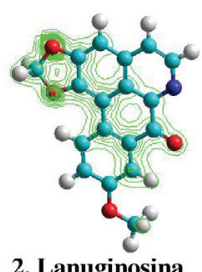

2. Lanuginosina

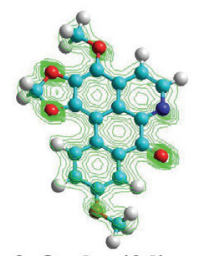

3. Oxobuxifolina
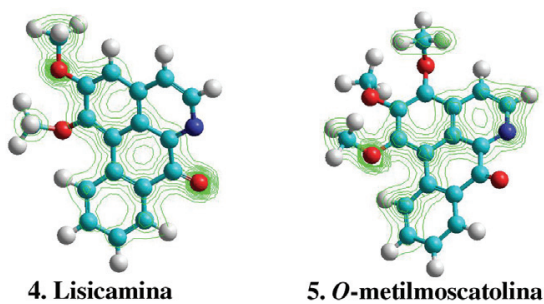

Figura 3. Densidade de carga total dos alcalóides oxaporfínicos apresentadas através das distribuições das nuvens eletrônicas sobre os átomos das moléculas

Foi possível também otimizar e analisar ${ }^{18}$ as propriedades eletrônicas e termodinâmicas. Ficou demonstrado através do cálculo teórico ab initio, que é mais quantitativo, que a afinidade protônica é praticamente a mesma $\left(-250 \mathrm{kcal} \mathrm{mol}^{-1}\right)$ para todos os alcalóides, exceto $O$-metilmoscatolina, com valor um pouco menor $(-243,2 \mathrm{kcal}$ $\mathrm{mol}^{-1}$ ), mostrando uma menor liberação de energia ao ser protonada.

\section{Atividade citotóxica}

As atividades biológicas e farmacológicas de alcalóides aporfinóides constituem um tema de avaliação e discussão, sendo bastante exploradas as potencialidades desses compostos como agentes citotóxicos e antitumorais. A relação estrutura-atividade dos aporfinóides geralmente envolve o padrão de substituição $0^{2,3} \mathrm{e}$, em especial, quando se consideram os oxaporfínicos, a planaridade da molécula com relação aos demais alcalóides aporfinóides é motivo de destaque. Como já mencio- nado, há diferença na interação direta sobre o DNA de alcalóides aporfínicos e oxaporfínicos, o que é exemplificado pela dicentrina e liriodenina, respectivamente, sendo que, para esta última sugere-se ser mais eficiente devido a sua estrutura mais planar $^{8}$. A planaridade dos oxaporfínicos ocorre em função da carbonila em C-7, que favorece a extensão da conjugação no sistema aporfínico.

O padrão de substituição no anel A é considerado fundamental. Por exemplo, a inserção de substituintes do tipo 1,2-metilenodioxila em alcalóides oxaporfínicos contribui para a atividade citotóxica desses alcalóides, embora também tenha sido demonstrada uma baixa seletividade, como ocorre com a liriodenina, enquanto a asteropermidina, com substituintes em C-1, C-2 e C-3, é mais seletiva ${ }^{2}$.

No presente trabalho, dos seis alcalóides citados, quatro $(\mathbf{1}, \mathbf{2}, 4$ e 5) foram testados contra células $\mathrm{Hep}_{2}$ (ATCC-CCL 23, de carcinoma de laringe), as quais se mostraram mais sensíveis ao alcalóide $\mathbf{5}$, que apresentou menor caráter básico. A Figura 3 mostra a densidade de carga total dos alcalóides oxaporfínicos, representada através das distribuições das nuvens eletrônicas sobre os átomos das moléculas, com maior densidade de carga sobre N-6 de 5, em comparação com os demais alcalóides. A $O$-metilmoscatolina poderia estar mais sujeita à ligação covalente que à iônica, o que poderia explicar o efeito citotóxico apresentado por esse alcalóide (Tabela 1).

Tabela 1. Efeito citotóxico $\left(\mathrm{CI}_{50}\right)$ em $\mu \mathrm{M}$ dos alcalóides oxaporfínicos em células $\mathrm{Hep}_{2}$ em $24 \mathrm{~h}$.

\begin{tabular}{lcc}
\hline Substâncias & $\mathrm{CI}_{50}$ & $\begin{array}{c}\text { Intervalo de } \\
\text { confiança 95\% } \mu \mathrm{M}\end{array}$ \\
\hline Liriodenina (1) & 44,3 & $39,1-50,3$ \\
Lanuginosina (2) & 61,1 & $55,7-67,1$ \\
Lisicamina (4) & 103,6 & $97,5-110,1$ \\
$O$-metilmoscatolina (5) & 12,4 & $9,3-16,6$ \\
Cisplatina & 18,0 & $13,9-23,5$
\end{tabular}

$\mathrm{CI}_{50}$ : dose eficaz que inibe $50 \%$ do crescimento celular $(\mu \mathrm{M})(\mathrm{n}=3)$; Probit Analysis.

\section{PARTE EXPERIMENTAL}

\section{Marcha química para alcalóides}

O extrato alcaloídico de cada espécie vegetal foi obtido da maneira usual, ou seja, $100 \mathrm{~g}$ de planta foram submetidas à maceração em $\mathrm{NH}_{4} \mathrm{OH}$ a $10 \%$, seguida da adição de $\mathrm{CHCl}_{3}$, com volume suficiente para extração e mantido em repouso por $72 \mathrm{~h}$. Após a decantação, procedeu-se na extração com solução de ácido clorídrico $5 \%$ e, posteriormente, a camada ácida obtida foi ajustada até $\mathrm{pH} 8,0$ e novamente extraída com $\mathrm{CHCl}_{3}$. Os extratos alcaloídicos obtidos foram solubilizados em clorofórmio e submetidos à partição com soluções 5\% de diferentes ácidos (com variação de pKa): cítrico, acético e oxálico.

\section{Cromatografia em camada preparativa de sílica-gel impregnada com ácido oxálico}

Inicialmente, as placas cromatográficas foram confeccionadas com sílica-gel e solução 5\% dos ácidos cítrico, acético e oxálico. Para a produção de uma placa preparativa $20 \mathrm{~cm} \times 20 \mathrm{~cm}$, com espessura de 1,0 mm, utilizaram-se $20 \mathrm{~g}$ de sílica-gel 60G, $2 \mathrm{~g}$ de sílica-gel GF254 (Type 60) (ambas Merck) e 55 mL de solução desses ácidos a $5 \%$. As placas foram mantidas em local protegido de correntes de ar e de luz por $24 \mathrm{~h}$ para secagem e posteriormente foram ativadas em 
estufa a $110{ }^{\circ} \mathrm{C}$ por $1 \mathrm{~h}$. (Cuidado! Vapores tóxicos.)

$\mathrm{O}$ mesmo procedimento foi utilizado nas placas de sílica-gel impregnadas com diferentes concentrações de ácido oxálico, sendo mais eficazes para a separação dos alcalóides oxaporfínicos aquelas que apresentaram concentração de $12,1 \%$ p/p.

\section{Cromatografia em coluna}

Uma mistura dos alcalóides $\mathbf{1}$ e $\mathbf{6}(15 \mathrm{mg})$ foi submetida a nova separação em sílica-gel (25 g), utilizando como eluente $\mathrm{CHCl}_{3}: \mathrm{MeOH}$ em gradiente de polaridade, juntamente com $0,5 \%$ de $\mathrm{HCl}$ concentrado. Foram recolhidas 22 frações, verificando-se que as frações 5-8 (3 mg) consistiam no alcalóide aterospermidina (6) e as frações 19-21 em liriodenina (1) (7 mg).

Liriodenina (1): 7,0 mg, sólido cristalino amarelo, com baixa solubilidade em $\mathrm{CHCl}_{3}$. Os dados de ${ }^{1} \mathrm{H} \mathrm{RMN}$ (300 MHz, $\mathrm{CDCl}_{3}$ ) estão de acordo com os descritos na literatura ${ }^{16}$.

Lanuginosina (2): 9,2 mg, sólido cristalino alaranjado, com baixa solubilidade em $\mathrm{CHCl}_{3}$. Os dados de $\mathrm{RMN}$ de ${ }^{1} \mathrm{H}(300 \mathrm{MHz}$, $\mathrm{CDCl}_{3}$ ) estão de acordo com os descritos na literatura ${ }^{16}$.

Oxobuxifolina (3): 2,0 mg, sólido amarelo-escuro. Os dados espectrais de $\mathrm{RMN}$ de ${ }^{1} \mathrm{H}\left(300 \mathrm{MHz}, \mathrm{CDCl}_{3}\right.$ ) estão de acordo com os descritos na literatura ${ }^{19}$.

Lisicamina (4): 7,0 mg, sólido cristalino amarelo, com baixa solubilidade em $\mathrm{CHCl}_{3}$. Seus dados de $\mathrm{RMN}$ de ${ }^{1} \mathrm{H}(300 \mathrm{MHz}$, $\mathrm{CDCl}_{3}$ ) foram confirmados pelos descritos na literatura ${ }^{19}$.

$O$-metilmoscatolina (5): $10,3 \mathrm{mg}$, sólido cristalino alaranjado, solúvel em $\mathrm{CHCl}_{3}, \mathrm{RMN}$ de ${ }^{1} \mathrm{H}\left(300 \mathrm{MHz}, \mathrm{CDCl}_{3}\right): \delta 9,09(1 \mathrm{H}, \mathrm{d}$, $J=8,0 \mathrm{~Hz}, \mathrm{H}-11), 8,95(1 \mathrm{H}, \mathrm{d}, J=5,3 \mathrm{~Hz}, \mathrm{H}-5), 8,56(1 \mathrm{H}, \mathrm{d}, J=8,0$ $\mathrm{Hz}, \mathrm{H}-8), 8,20$ (1H, d, $J=5,3 \mathrm{~Hz}, \mathrm{H}-4), 7,73$ (1H, m, H-10), 7,53 $(1 \mathrm{H}, \mathrm{m}, \mathrm{H}-9), 4,18$ (3H,s, $\mathrm{OCH}_{3}$ em C-3), 4.09 (3H,s, $\mathrm{OCH}_{3}$ em C1), $4,0\left(3 \mathrm{H}, \mathrm{s}, \mathrm{OCH}_{3} \text { em C-2 }\right)^{20}$.

Aterospermidina (6): $3,0 \mathrm{mg}$, cristais alaranjados. Os dados espectrais de $\mathrm{RMN}{ }^{1} \mathrm{H}\left(300 \mathrm{MHz}, \mathrm{CDCl}_{3}\right)$ estão de acordo com os descritos na literatura ${ }^{16}$.

\section{Ensaio de citotoxicidade em linhagens de células tumorais}

A inibição do crescimento celular, um indicador da citotoxicidade causada pelas substâncias que estão sendo avaliadas quanto à atividade antineoplásica, foi medida utilizando o método MTT [3-(4,5dimethylthiazol-2-yl)-2,5-diphenyltetrazolium bromide], essencialmente conforme descrito ${ }^{20}$. Este é um sal solúvel de tetrazólio, e somente células vivas são capazes de reduzí-lo a um produto colorido insolúvel, o formazan, que pode ser medido após sua dissolução com DMSO.

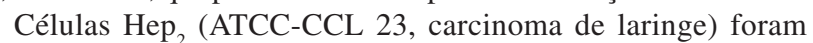
semeadas em placas de 96 cavidades (100 000 células/mL) e mantidas em meio de cultura DMEM a $37{ }^{\circ} \mathrm{C}$ em incubadora com $5 \%$ de $\mathrm{CO}_{2}$. Após $24 \mathrm{~h}$ o meio foi removido para adição das substâncias-teste e do controle positivo (cisplatina, Sigma). A concentração de cada substância variou de 1 a $50 \mu \mathrm{g} / \mathrm{mL}$, sendo cada uma da quatro concentrações adicionadas em triplicata. Ao final da exposição de $24 \mathrm{~h}$, o meio foi substituído por meio de cultura fresco contendo MTT (concentração final: $1,0 \mathrm{mg} / \mathrm{mL}$ ) e as placas foram incubadas por mais $4 \mathrm{~h}$.

Ao final, o produto formazan foi dissolvido em DMSO durante 10 min e a absorbância foi determinada a $570 \mathrm{~nm}$ com leitor de microplacas (Rosys Anthos 2001).

A concentração que inibe $50 \%$ do crescimento celular $\left(\mathrm{CI}_{50}\right)$ foi calculada com o programa Probitos ${ }^{21}$, a partir das diferenças de leitura de absorbância entre células não-tratadas (controle negativo) e tratadas (substância-teste).

\section{AGRADECIMENTOS}

À FUNDECT/MS e PROPP-UFMS, pelo financiamento do projeto. As bolsas foram fornecidas pelo FUNDECT-CNPq. À R. R. de Oliveira (bolsista de apoio técnico, $\mathrm{CNPq}$ ), pelo suporte técnico durante a parte experimental do estudo. À Agência Estadual de Defesa Sanitária Animal e Vegetal (IAGRO) e ao Departamento de Morfofisiologia, CCBS, UFMS, por cederem os equipamentos. Ao Prof. Dr. A. E. da H. Machado, do Laboratório de Fotoquímica, UFU, por disponibilizar o pacote Hyperchem para geração das figuras.

\section{REFERÊNCIAS}

1. Cavé, A.; Leboeuf, M.; Waterman, P. G. Em Alkaloids: Chemical and Biological Perspectives; Pelletier. S. W., ed.; John Wiley \& Sons: Nova Iorque, 1987, vol. 5; Leboeuf, M.; Cavé, A.; Bhaumik, P. K.; Mukherjee, B.; Mukherjee, R.; Phytochemistry 1982, 21, 2783.

2. Stévigny, C.; Bailly, C.; Quentin-Leclercq, J.; Curr. Med. Chem. 2005, 5, 173.

3. Boustie, J.; Stigliani, J-L.; Montanha, J.; Amoros, M.; Payard, M.; Girre, L.; J. Nat. Prod. 1998, 61, 480.

4. Sonnet, P. E.; Jacobson, M.; J. Pharm. Sci. 1971, 60, 1254; Wu, Y. C.; Chen, C. H.; Yang, T. H.; Lu, S. T.; McPhail, D. R.; McPhail, A. T.; Lee, K. H.; Phytochemistry 1989, 28, 2191; Wijeratne, E. M. K.; Gunatilaka, A. A. L.; Kingston, D. G. I.; Haltiwanger, R. C.; Eggleston, D. S.; Tetrahedron 1995, 51, 7877; Castro, I.; Villegas, J. R.; Soeder, B.; Castro, O.; Fitoterapia 1996, 67, 181; Guinaudeau, H.; Leboeuf, M.; Cavé, M.; J. Nat. Prod. 1975, 38, 275.

5. Chen, K. S.; Ko, F. N.; Teng, C. M.; Wu, Y. C.; Planta Med. 1996, 62, 133.

6. Hufford, C. D.; Funderburk, M. J.; Morgan, J. M.; Robertson, L. W.; J. Pharm. Sci. 1975, 64, 789; Hufford, C. D.; Sharma, A. S.; Oguntimein, B. O.; J. Pharm. Sci. 1980, 69, 1180; Nieto, M.; Cavé, A.; Leboeuf, M.; J. Nat. Prod. 1976, 39, 350.

7. Barreiro, E. J.; Fraga, C. A. M.; Química Medicinal: As Bases Moleculares da Ação de Fármacos, $1^{\mathrm{a}}$ ed., Artmed: Porto Alegre, 2002.

8. Woo, S. H.; Reynolds, M. C.; Sun, N. J.; Cassady, J. M.; Snapka, R. M.; Biochem. Pharmacol. 1997, 54, 467; Woo, S. H.; Sun, N. J.; Cassady, J. M.; Snapka, R. M.; Biochem. Pharmacol. 1999, 57, 1141.

9. Wijeratne, E. M. K.; Hatanaka, Y.; Kikuchi, T.; Tezuka, Y.; Gunatilaka, A. A. L.; Phytochemistry 1996, 42, 1703.

10. Wu, Y. C.; Chang, G. Y.; Duh, C. Y.; Wang, S. K.; Phytochemistry 1993, $33,497$.

11. de Siqueira, J. M.; Ziminiani, M. G.; Resende, U. M.; Boaventura, M. A. D.; Quim. Nova 2001, 24, 185; de Siqueira, J. M.; Bomm, M. D.; Pereira; N. F. G.; Garcez; W. S.; Boaventura, M. A. D.; Quim. Nova 1998, 21, 557.

12. Stahl, E.; Thin-Layer Chromatography: A Laboratory Handbook, $6^{\mathrm{a}}$ reimp. da $2^{\text {a }}$ ed., Springer-Verlag: New York, 1990; Wagner, H.; Bladt, S.; Plant Drug Analysis: A Thin Layer Chromatography Atlas, $2^{\text {nd }}$ ed., Springer: New York, 1996.

13. Djilani, A.; Legseir, B.; Soulimani, R.; Dicko, A.; Younos, C.; J. Braz. Chem. Soc. 2006, 17, 518.

14. Paris, M.; Hurabielle, M.; Abrégé de Matière Médicale (Pharmacgnosie)Tome I : Généralités - Monographies $1^{\text {re }}$ Partie, Ed. Masson: Paris, 1981, p. 261-265; Henriques, A. T.; Limberger, R. P.; Kerber, V. A.; Moreno, P. R. H. Em Farmacognosia da Planta ao Medicamento; Simões, C. M. O.; Schenckel, E. P.; Gosmann, G.; de Mello, J. C. P.; Mentz, L. A.; Petrovick, P. R., orgs.; 5 a ed., UFSC \& UFRGS: Florianópolis Porto Alegre, 2003.

15. Schulman, S. G.; Capomacchia, A. C.; J. Am. Chem. Soc. 1973, 95, 2763.

16. Guinaudeau, H.; Leboeuf, M.; Cavé, A.; J. Nat. Prod. 1994, 57, 1033; Guinaudeau, H.; Leboeuf, M.; Cavé, A.; J. Nat. Prod. 1988, 51, 389; Guinaudeau, H.; Leboeuf, M.; Cavé, A.; J. Nat. Prod. 1975, 38, 304.

17. Zielinski, W.; Kudelko, A.; Arkivoc 2005, 5, 66.

18. Güven, A.; Ögretir, C.; J. Molec. Struct. (Theochem) 1998, 434, 7; Kallies, B.; Mitzner, R.; J. Molec. Struct. (Theochem) 1998, 428, 267; Tran, N. L.; Colvin, M. E.; J Molec. Struct. (Theochem) 2000, 532, 127.

19. Harrigan, G. G.; Gunatilaka, A. A. L.; Kingston, D. G. I.; Chan, G. W.; Johnson, R. K.; J. Nat. Prod. 1994, 57, 68.

20. Mosmann, T.; J. Immunol. Methods. 1983, 65, 55.

21. Finney, D. J.; Probit Analysis, Cambridge University Press: Cambridge, $1971,3^{\text {rd }}$ ed. 